\title{
A Review on Study of Sugar for its Market Growth, Processing Chemistry and Comparison with Other Sweeteners
}

\author{
Bandana Chatterjee Flourish* \\ Department of Research and Development, Flourish Pure foods Pvt Ltd., India \\ *Corresponding author: Bandana Chatterjee Flourish, Department of Research and Development, Flourish Pure foods Pvt. Ltd, India
} Submission: April 17, 2018; Published: May 23, 2018

\begin{abstract}
Despite an expected fall, global sugar consumption should rise during the remaining decade to sustain the global demand and reduction in stocks. The sugar production is foreseen an average growth of $2.1 \%$. With this rise, sugarcane is expected to account for $86 \%$ mass production of sugar and remaining is to be accounted by sugar beet. Sugar is a carbohydrate, named sucrose, mostly found in all fruits \& vegetables. Green plants uses photosynthesis process to prepare carbohydrate and then, plants converts them into energy source and also store it. A stalk of the cane plant contains about $14 \%$ sugar. Sugar beets contain about $16 \%$ sugar. Depend upon the availability of raw material, the process to prepare sugar differs and various by-products are also prepared at different stages depending upon their application in food sector.

Carbohydrates are polyhydroxy aldehydes or ketones or compounds which breaks down into smaller units on hydrolysis. Depending upon the sugar molecules, the sacchrides are classified as: mono sacchrides, disaccharides, oligosaccharides and polysaccharides. Sugar is a high calorie carbohydrates, but there are various other types of sweeteners used in food industry like dextrose, high fructose corn syrup etc.
\end{abstract}

\section{Market Scenario}

Sugar industry is one of the few industries having growth and that has been contributing successfully towards the growth of rural economy. This growth majorly depends upon the availability and utilisation of raw material used for the production to meet the increase demand of sugar in India and also to support the Indian economy. Indian sugar industry plays an important role in rural development. The industry helps a lot of farmers and their families as the source required to produce sugar are sugarcane and sugar beet, which is also the sole income source for these farmers. Globally, the key market of sugar like Brazil \& Thailand, gets affected by various regulations imposed on the sector. There are various reasons of these regulations, but the main factors are perishable nature of cane, small farm landholdings, prices etc. Sugar is regulated in India too, especially due to climatic conditions and losses occur due to improper supply chain. Since 1993, the environmental factor has not been much of an issue, but sugar is still an important commodity for consumers on a regular basis.

Despite an expected fall in the coming season in certain sugar production countries, the sugar production globally tends to rise during the next decade, with an intention to meet the growing demand and maintain the stocks. It is expected that the growth in production of sugar during that decade to increase by $2.1 \%$ per annum (p.a.), reaching a production of 210 Mt by 2025, i.e. an increase of about $39 \mathrm{Mt}$ over the base period (2013-2015). The major proportion of sugar produced is generated from sugar cane instead of sugar beet. This increase in production of sugar over the next decade will majorly come from the sugarcane producing countries instead of those produces sugar beet. The other factor that will play a crucial role are area expansion and yield improvement in countries like India, Thailand etc.

The raw materials used to produce sugar, are cane and beet, of which sugarcane will account for about $86 \%$, while the remaining $14 \%$ is covered by sugar beet over the next decade. Major countries participating in sugar production from sugar beet are Russian Federation, Egypt, Ukraine and so on. As stated earlier, the main factors influencing the production of sugar are area expansion and yields improvement. Certain countries uses the waste generated after sugar production to convert them as use as an energy source. Some share of sugarcane produce is also used for the production of ethanol as a fuel and its trend tends to increase and is expected that about $22 \%$ of sugarcane in 2025 will be allocated to produce ethanol. 


\section{Introduction}

Sugar is sucrose, which is a carbohydrate, and is found in almost all fruits and vegetables. All green plants used the process of photosynthesis, in order to transfer the direct sunlight into food i.e. sugar and as a source of energy. This sugar is, then, converted into starch and then into different forms of sugar to store for their use in future. This function of plants of converting starch into different forms of sugar helps in providing a wide range of tasty fruits and vegetables, ranging from mango to sweet carrot. The main sources to produce sugar are sugarcane and sugar beet, and are well known for the commercial production of sugar. According to research, a stalk of sugarcane contains about $14 \%$ sugar, while that of sugar beet contains $16 \%$ sugar content. The sugar that is extracted from the source has identical chemical structure. The taste, colour, smell and behaviours of sugar obtained from sugarcane and sugar beet are similar. Sugar that is either used as an ingredient or sweetner at home is $99.9+\%$ sucrose.

The sweetner which is used to compare the sweetness is sucrose and is considered as a standard to measure the relative sweetness of other sweetners. The only way to determine or measure the sweetness is by taste it. Whenever, the substance is tasted and touched to the tongue, the taste buds send the signals to the brain, where the brain identifies and determine the sweetness. The most common sweetners are glucose, sucrose and fructose. As far as the sweetness is concerned, glucose is sweeter than sucrose, which itself is sweeter than fructose. Sugar can either be present in food item naturally or can be added to food externally as a carbohydrate depending upon the requirement and plays an important in all the food items. Sugar as carbohydrates are considered to be a source of energy and also act as a sweetner. Presence of sugar provide taste to food products both processed or unprocessed. Certain recent studies shows a relation between the consumption of sugar and health of customers, depending upon various factors like the intake of sugar, type of sugar etc.

Almost everyone enjoys sugars and sweets, but many consumers wonder whether consumption of sugars affects health. This paper represents production of sugar, molecular structure of sugar, nutrition and health aspects of sugars consumption, summarizes nutrition and policy recommendations of the scientific community.

The objective of this paper are as follows:

a) A fully review on sugar right from the commercial source to the production of final finished product; and

b) Benefits \& limitations of sugar consumption in a day to day life.

\section{Production of sugar}

Major commercial source presently available are sugarcane and sugar beet. The process to prepare sugar varies with source. For most people, the term "refine" has a negative meaning, but it's actual meaning is "to purify i.e. to remove the impurities or unwanted material". With reference to sugar refining, this term means the removal of impurities and particulated matter, leaving pure sucrose. The detailed general process of preparing sucrose (sugar) is given as follows:

1. Washing, Cutting and Crushing

2. Clarification

3. Evaporation

4. Crystallization

5. Mixing

6. Centrifugation

7. Evaporation

8. Storage

Washing, cutting and crushing: At initial stage, sugarcane stalks are cleaned using water and then cut into shreds by rotating knives. After cutting, huge rollers presses the cutted shreds to extract the juice.

Clarification: Now, the juice extracted is mixed with lime solution and carbon dioxide. The carbon dioxide reacts and bubbled through water forming calcium carbonate, which has the property to attract impurities present in juice like wax, fats etc. In clarifier, the impurities from juice along with calcium carbonate settles at bottom leaving sucrose solution.

Evaporation: In an evaporator, the clarified juice obtained from clarifier is heated under vacuum in multiple stages. The vacuum during heating clarified juice helps in evaporating water present at lower temperature and thus preventing from caramalization. Then, the juice becomes clear and rich brown syrup.

Crystallization: On further heating the brown syrup under vacuum, the water is evaporated leaving the raw sugar and then tends to form crystals as the last portion of water evaporates. The mixture is, then, transfered from vacuum pan to centrifuge, a perforated basket rotating at high speed in a spin cycle, and drying the mixture and providing the golden yellow sugar.

The raw sugar obtained above is about 96-98\% sucrose (approx.). This nearly pure sugar is covered with a thin layer of molasses solution, which itself composed of sugar, water, and some impurities.

Mixing: This obtained raw sugar is then transfered to the refinary, where it is mixed with warm mixture of water and sugar. This syrup mixture will help in loosening the molasses layer from the raw sugar. Thus, the mixture obtained is known as magma.

Centrifugation: Now, the mixture magma obtained transfered to a cylinderical container, which rotates at very high speed, seperating the molasses film from the crystals. Then, the crystals obtained are transfered through a splash of water to clean, and dissolve the molasses layer in water and seperating the molasses via filtering or clarifying. The product obtained here is completely 
clear golden coloured sucrose solution. This solution is now passes through carbon filter, producing a white coloured sucrose syrup. Now, this syrup is tranfered to a evaporator.

Evaporation: The white coloured sucrose syrup is then heated in evaporator under vacuum conditions. Then, with proper evaporation, water is removes and the syrup gets converted into fine sugar crystals of proper paticle size.

Storage: At this final stage, the sugar crystals are washed with hot water and is further spun using centrifugal force in order to remove any moisture and impurities present with crystals. The moisture of sugar crystals is reduced to $1 \%$ to $0.3 \%$ to store crystals. Being hygroscopic in nature, the crystals have the tendency to absorb moisture from surrounding, so is kept in dry conditions in properly packed bags or containers.

Before storing the crystals, the dried crystals allows to pass through number of screens to grade the sugar and are stored separately depending upon the grading and its application in food industry.

\section{Types of sugar}

Raw sugar: Raw sugar is prepared inset as an intermediate product during the production of sugar. It can be identified as a coarse granulated product, produced at the end of milling process after the evaporation of clarified cane sugar syrup. This raw sugar is transfered for refinery for final processing to prapare sugar.

Brown sugar: The sugar obtained at forth stage of sugar preparation, explained in processing of sugar is termed as Brown sugar. Brown sugar means the raw sugar crystals coated with molasses film, which provide the natural colour and flavor to crystals. Some manufacturers produces brown sugar by boiling molasses syrup till brown sugar crystals forms. The centrifugal force is applied to the crystals formed to dry and remove impurities.

Turbinado sugar: This sugar is prepared after the raw sugar is transfered for refinery. The type of sugar which is prepared after removing the molasses layer by using the washing and centrifugation process. This type of sugar is even more pure than the raw sugar.

Molasses: The remaining liquid syrup left during refining stage, where the raw sugar is refined to produce sucrose. The extraction of molasses involves 3 steps, and at each step, different type of molasses is produced as follows:

1. Light molasses is produced at the first stage, is sweeter than the other two, but has the mildest flavor. It is used in food products like cookies, cakes etc.

2. Dark molasses is prepared during stage, is less sweet and has better flavor than previous one. It is most often used in baked beans.

3. Blackstrap molasses is prepared at final stage, has least sweetness and more flavor than the previous two. It has a common application in animal feed.
The molasses prepared from sugar beet has application in livestock feed and as commercial ingredient to produce industrial yeast. The molasses syrup available in market is prepared by blending molasses, prepared during sugar refining, with sugar solution to ensure the taste, flavour and quality.

Invert sugar: Invert sugar is a liquid carbohydrate sweetener in which all or a portion of the sucrose present has been inverted: the sucrose molecule is split and converted to a mixture of glucose and fructose. Invert sugars help baked goods retain moisture and prolong shelf-life. Candy manufacturers use invert sugar to control graining.

\section{Chemistry of sugar}

Carbohydrates are polyhydroxy aldehydes or ketones or compounds which breaks down into smaller units (known as monosaccharides) on hydrolysis. Carbohydrates are the most abundant source of energy and participate in structure of cell membrane and cellular functions like cell growth, fertilization etc. Carbohydrates, based on number on sugar units present, are classified as:

1. Monosaccharide's - Cannot be hydrolyzed further into simpler forms.

2. Disaccharides - Yield 2 molecules of same or different monosaccharide units on hydrolysis.

3. Oligosaccharides-Yield 3-10 molecules of monosaccharide units on hydrolysis.

4. Polysaccharides - Yield more than 10 molecules of same or different monosaccharide units on hydrolysis.

And the smallest units generate after hydrolysis is shown as Figure 1.

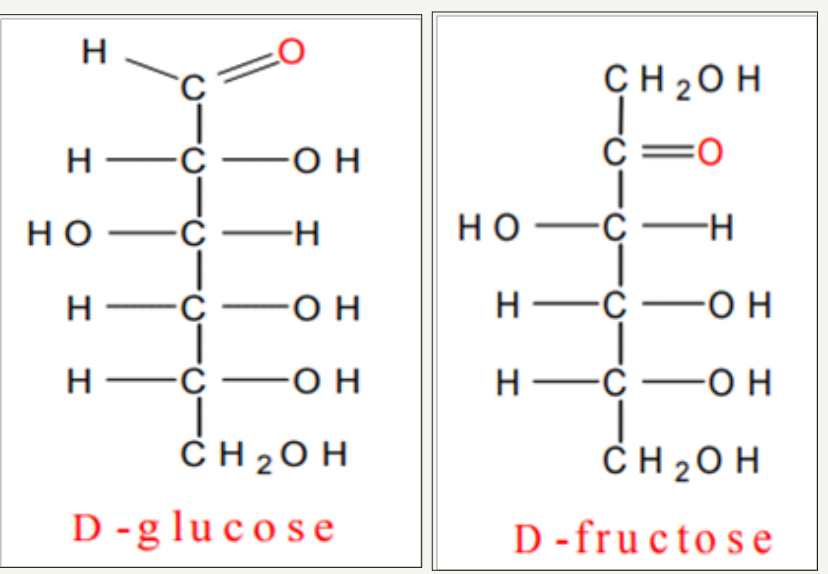

Figure 1: The smallest units generate after hydrolysis.

As stated earlier, sugar also known as sacchrides, is the most easily available and abundantly available molecule. The sacchrides are of different types and each has different application. Sugar is available in most of the foods as an ingredient. Sacchrides also functions as structural material in cell walls of plants and bacteria 
and in the connective tissue and cell coats of animals. These molecules also act as a lubricant at muscle joints. Also, sugar has the ability to get attached with protein (in glycoproteins) and lipids (in glycolipids) and these compounds act as (i) as antigenic sites; (ii) provide signals that determine the cellular localization of proteins and (iii) function as signals that allow cells to recognize each other and adhere in the formation of tissues and organs.

Monosaccharides: "Monosaccharides" term belongs to greek terminology "Mono" means single and "sacchrides" means sugar. These are polyhydroxy aldehydes or ketones, which cannot be break down into smaller components. They are crystalline in nature and soluble in water. The structure of monosaccharide's may contain carbon atoms (3 to 10), hydroxyl $(\mathrm{OH})$ groups (2 or more) and either a aldehydic ( $\mathrm{CHO}$ ) or a ketonic ( $\mathrm{CO}$ ) group. Some of the monosaccharide's are shown in Table 1.

Table 1: Some of the Mono saccharides.

\begin{tabular}{|c|c|c|c|}
\hline Name & Formula & Aldose & Ketose \\
\hline Terose & $\mathrm{C}_{4} \mathrm{H}_{8} \mathrm{O}_{4}$ & Erythrose & Erythrulose \\
\hline Pentose & $\mathrm{C}_{5} \mathrm{H}_{10} \mathrm{O}_{5}$ & Ribose & Ribulose \\
\hline Hexose & $\mathrm{C}_{6} \mathrm{H}_{12} \mathrm{O}_{6}$ & Glucose & Fructose \\
\hline Heptose & $\mathrm{C}_{7} \mathrm{H}_{14} \mathrm{O}_{7}$ & Glucoheptose & Sedo heptulose \\
\hline
\end{tabular}

1. Straight or open chain structure: In this, carbon atoms of a chain are arranged in a manner to be appeared in straight line. It is also called open chain structure as the two ends of chain remain separate and they are not linked.

2. Cyclic or ring structure: Here the extreme atoms of chain are arranged and linked and forms a ring.

Di-, Oligo- \& Poly-Sacchrides: The biochemical reaction that represents the condensation (i.e. removal of water) of two (or more) monosaccharides from 2 - $\mathrm{OH}$ groups present on two sugar If the reaction involves 2 monosaccharides, then it is Di-sacchrides; if the reaction involves 3-10 monosaccharides, then it is Oligosacchrides; while involving more than 10 monosaccharides results in polysaccharides. The reaction between two or more than two monosaccharides, occurs between $\mathrm{OH}$ group present at $\mathrm{C} 1$ of one monosaccharide with that present on $\mathrm{C} 4$ of the other. The bond formed during linkage is termed as Glucosidic Bond.

Polysaccharides are many monosaccharides linked by glycosidic bonds. Most poly-saccharides are polymers of either glucose, or modified glucose. They play important roles in the structure of plants (cellulose) as well as energy storage in both plants (starch) and animals (glycogen)

\section{Other carbohydrate sweeteners}

Honey: Honey is a sweet and viscous food substance, majorly contains sugar that is formed from an enzyme, invertase that is present in bees bodies. The colour, flavor and quality of honey mainly depend upon the source, from which the nectar is extracted. The compositional value of honey consists of fructose (about 38\%), glucose (about $31 \%$ ), sucrose (about 1\%), other sugar (9\%), water $(17 \%)$ and ash content $(0.17 \%)$.
High fructose corn syrup (HFCS): High fructose corn syrup act as a sweetner, produced from corn starch by treating the same with glucose isomerase (enzyme), which converts some of the glucose into fructose. The standard HFCS compound mainly composed of fructose (composition varies from 42-99\%) and glucose.

High fructose corn syrup (HFCS) has a wide range of application in food industry like soft drinks, canned fruits, jams etc., but also has certain negative health concerns like increase risk of obesity, increased risk of type- 2 diabetes.

Dextrose: Dextrose is chemically identical to glucose, crystalline in structure, and is prepared from starch. It acts as a sweetner in baked products, desserts like custards, sherbets etc. and processed foods like cookies etc. Dextrose is $60-70 \%$ as sweet as sugar and has around 4 calories per gram.

Crystalline fructose: The fructose enriched corn syrup is used to prepare crystalline fructose by using the process of crystallization. The crystallized fructose, having an application in food industry as an ingredient in foods and beverages, is prepared from corn starch. It can be considered as nearly pure fructose and is more sweeter than sugar. The crystalline sugar has the similar application as that of high fructose corn syrup e.g. Soft drink, canned fruits etc.

Maltodextrin: Potato, corn and rice are the main source used to prepare maltodextrin. It is a non-sweetener, energy source i.e. 4 calorie per gram, and thus, like other sweeteners, is not necessarily need to be mentioned among ingredients.

Maltodextrins has a wide range of applications in food industry, right from canned fruits to snacks and can also be used as an ingredient in table top single serve artificial sweetners. Maltodextrin also has an application as bulking agent and thus, provide texture to food.

Fructo-oligosaccharides: Fructo-oligosaccharides (FOS) are oligosaccharides that has been naturally occured in different plant source such as onion, garlic asparagus, chicory etc. FOS is derived from food grade sucrose via a transfructosylation catalyzed by Pfructofuranosidase enzyme derived from a non-pathogenic and non-toxigenic strain of Aspergillus oryzae. Fructooligosaccharides (FOS) are a mixture of oligosaccharides consisting of a sucrose molecule (glucose - fructose disaccharide, GF1) linked to one (GF2), or two (GF3) or three (GF4) additional fructose units added by P2-1 glycosidic linkages to the fructose unit of the sucrose. Fructans can have degrees of polymerization (the number of fructose or glucose residues) ranging from 2 to over 60 [1-15].

\section{References}

1. Li Y, Schellhorn HE (2007) New developments and novel therapeutic perspectives for vitamin C. Nutr 137(10): 2171-2184.

2. Carr AC, Frei B (1999) Toward a new recommended dietary allowance for vitamin $\mathrm{C}$ based on antioxidant and health effects in humans. The American journal of clinical nutrition 69(6): 1086-1107.

3. Frei B, England L, Ames BN (1989) Ascorbate is an outstanding antioxidant in human blood plasma. Proc Natl Acad Sci U S A 86(16): 6377-6381. 
4. Jacob RA, Sotoudeh G (2002) Vitamin C function and status in chronic disease. Nutr Clin Care 5(2): 66-74.

5. Gershoff SN (1993) Vitamin C (ascorbic acid): new roles, new requirements? Nutrition reviews 51(11): 313-326.

6. Institute of Medicine, Food and Nutrition Board (2000) Dietary reference intakes for vitamin C, vitamin E, Selenium and Carotenoids. National Academy Press, Washington, DC, USA.

7. Stephen R, Utecht $T$ (2001) Scurvy identified in the emergency department: a case report. J Emerg Med 21(3): 235-237.

8. Weinstein M, Babyn P, Zlotkin S (2001) An orange a day keeps the doctor away: scurvy in the year 2000. Pediatrics 108(3): e55-e55.

9. Wang AH, Still C (2007) Old world meets modern: a case report of scurvy. Nutrition in Clinical Practice 22(4): 445-448.

10. Mayo Clinic (2017) Ascorbic Acid (Oral Route). DRG-20068031 Drugs \& Supplements, Ascorbic Acid (Oral Route).

11. Aulton ME (2007) Aulton's Pharmaceutics: The Design and Manufacture of Medecines. ( $3^{\text {rd }}$ edn), Elsevier, Philadelphia, USA, pp. 145-151.

12. Chopra R, Alderborn G, Podczeck F, Newton JM (2002) The influence of pellet shape and surface properties on the drug release from uncoated and coated pellets. International journal of pharmaceutics 239(1): 171178.

13. Sadeghi F, Ford JL, Rajabi Siahboomi A (2003) The influence of drug type on the release profiles from Surelease-coated pellets. International journal of pharmaceutics 254(2): 123-135.
14. Lecomte F, Siepmann J, Walther M, MacRae RJ, Bodmeier R (2004) Polymer blends used for the aqueous coating of solid dosage forms: importance of the type of plasticizer. J Control Release 99(1): 1-13.

15. Aleksovski A, Spaseska Aleksovska E, Jašić, M (2012) Formulation and evaluation of ascorbic acid extended release hydrophilic matrix tablets by using hydroxypropyl methylcellulose and polyethylene oxide as matrix forming polymers. Hrana U Zdravlju I Bolesti 1(2): 22-31.

16. Pharma IQ, a division of IQPC (2017) Statistical Process Control (SPC). Pharmaceuticals \& Biotechnology Pharma \& Biotech Resources.

17. International Society for Pharmaceutical Engineering (ISPE) (2017) GAMP ${ }^{\circ}$ Good Practice Guide: GxP Compliant Laboratory Computerized Systems.

18. Minitab I (2014) MINITAB release 17: statistical software for windows. Minitab Inc., USA.

19. Shah S, Shridhar P, Gohil D (2010) Control chart: A statistical process control tool in pharmacy, Asian Journal of Pharmaceutics 4(3): 184.

20. Andreazza I, Ferraz H (2011) Preparation of pellets containing highly soluble drug by extrusion/spheronisation and coating with kollicoat $\AA$ SR 30D. Braz arch bio technol 54(2): 315-320.

21. Scibilia B (2015) A Simple guide to between / within capability.

22. (2016) Interpret the capability plot in capability sixpack Minitab.

23. Förmer P, Theurer C, Müller A, Schmidt PC (2006) Visualization and analysis of the release mechanism of shellac coated ascorbic acid pellets, Die Pharmazie. An International Journal of Pharmaceutical Sciences 61(12): 1005-1008.
Creative Commons Attribution 4.0 International License

For possible submissions Click Here

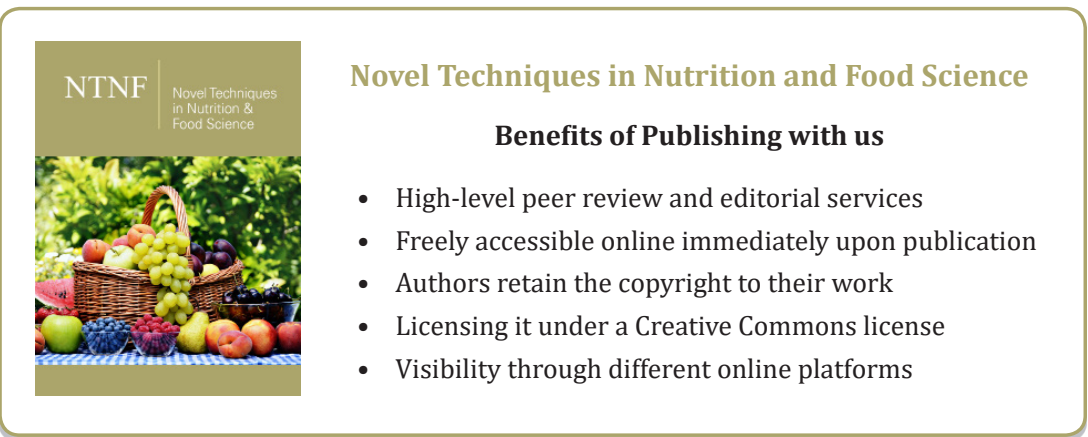

